\title{
EFFECT OF STORAGE TUBE MATERIAL AND RESVERATROL DURING LIQUID STORAGE OF MATURED BOVINE OOCYTES ON SUBSEQUENT DEVELOPMENT
}

\author{
Tayita SuttirojpattanA ${ }^{1,2}$, Tamás SomfaI ${ }^{2}$, Satoko MAtoBA $^{2}$, Takashi NAGAI $^{3}$, \\ Rangsun PARNPAI ${ }^{1}$ and Masaya GESHI ${ }^{2,4}$ \\ ${ }^{1}$ Embryo Technology and Stem Cell Research Center, Suranaree University of \\ Technology, Nakhon Ratchasima, Thailand; ${ }^{2}$ Animal Breeding and Reproduction \\ Research Division, Institute of Livestock and Grassland Science, National Agriculture \\ and Food Research Organization (NARO), Ikenodai 2, Tsukuba, Ibaraki 305-0901, \\ Japan; ${ }^{3}$ International Cooperation Section, National Agriculture and Food Research \\ Organization (NARO), Tsukuba, Japan; ${ }^{4}$ Division of Animal Sciences, Institute of \\ Agrobiological Sciences, National Agriculture and Food Research Organization \\ (NARO), Tsukuba, Japan
}

(Received 31 January 2017; accepted 6 November 2017)

This study determined the optimum storage vessel and the effects of resveratrol for the storage of in vitro matured (IVM) bovine oocytes. After IVM, the oocytes were kept in a Hepes-buffered medium at $25^{\circ} \mathrm{C}$ for $20 \mathrm{~h}$ in different containers including Eppendorf tubes (ET) made of polypropylene (PP) and polystyrene (PS), and tissue culture tubes (TCT) made of PP, PS, and glass. Then oocytes were subjected to IVF and subsequent in vitro embryo development was compared among the groups and to that of a control group without storage. The percentage of blastocyst development in the control group was significantly higher than in the stored groups $(\mathrm{P}<0.05)$. Among oocytes stored in TCT, the percentage of blastocyst development of oocytes stored in glass TCT was significantly higher than that of oocytes stored in PP and PS TCT $(\mathrm{P}<0.05)$; however, it did not differ from that of oocytes stored in ET. The quality of blastocysts did not differ among the control and stored groups. Embryo development was not affected when $0.1,1$ or $10 \mu \mathrm{M}$ resveratrol was added to the medium during oocyte storage. In conclusion, glass tubes were optimal for oocyte storage and resveratrol did not improve the development of stored oocytes.

Key words: Bovine, oocyte, storage, containers, resveratrol

In vitro embryo production (IVP) by in vitro oocyte maturation (IVM) and fertilisation (IVF) under laboratory conditions has become a potent method for the production of bovine embryos in large quantities using immature oocytes (Viana et al., 2010). However, approximately $5 \mathrm{~h}$ after the maturation is completed, the developmental competence of oocytes starts to decline due to oocyte

*Corresponding author; E-mail: somfai@affrc.go.jp; Phone/Fax: 0081 (298) 388-637 
ageing (Fissore et al., 2002). The optimal window period for IVF differs among species and has been determined for the bovine to be at 22-24 h of IVM (Long et al., 1994; Agung et al., 2006; Koyama et al., 2014). This fact appears to be a hitch for the planning of IVP programs when mature oocytes at the metaphase-II (MII) stage are obtained at awkward time points or spatially far from the site of IVF such as when ovum pick up (OPU) is performed at remote farms far from the laboratory. Therefore, preservation of matured oocytes without reducing their competence would be advantageous by making the planning of IVF programs more flexible in time and space. Cryopreservation of bovine oocytes is still difficult especially under field/farm conditions and it is associated with low embryo developmental rates (Mullen and Fahy, 2012). To overcome this problem, temporary storage of oocytes in media above the freezing point (i.e. liquid storage) offers an alternative for practical applications in the cattle industry.

We previously reported the production of good-quality blastocysts from IVM bovine oocytes stored for $20 \mathrm{~h}$ at $25^{\circ} \mathrm{C}$ in a HEPES-buffered medium in polypropylene Eppendorf tubes (Suttirojpattana et al., 2016). Nevertheless, blastocyst developmental rates remained lower than those obtained from fresh IVM oocytes. Previous studies suggest that the material of the vessel in which animal cells are kept might affect their development by toxicity (Hunter et al., 1988; Halldorsson et al., 2015). Furthermore, we have demonstrated that during liquid storage of bovine oocytes the intracellular levels of the antioxidant glutathione (GSH) decline, suggesting increased susceptibility to oxidative stress (Suttirojpattana et al., 2016). The aim of the present study was to assess the impacts of the storage vessel type/material and medium supplementation with the antioxidant resveratrol during liquid storage on the subsequent developmental competence of matured bovine oocytes.

\section{Materials and methods}

\section{Collection and IVM of bovine oocytes}

Bovine ovaries obtained at a slaughterhouse were kept and transported to the laboratory in plastic bags. After the bovine spongiform encephalopathy (BSE) test according to the Abattoir Law of Japan was confirmed to be negative, the ovaries were washed several times in $0.9 \%(\mathrm{w} / \mathrm{v}) \mathrm{NaCl}$ solution. Cumulusoocyte complexes (COCs) were aspirated from antral follicles $(2-8 \mathrm{~mm}$ in diameter) using a $10-\mathrm{ml}$ syringe with an 18-gauge needle, and only those surrounded by at least three layers of cumulus cells and with a homogenous cytoplasm were selected for IVM. Selected oocytes were washed three times in IVM medium, which was a HEPES-buffered TCM 199 medium (Medium 199, 12340-030, Gibco, Thermo Fisher Scientific, Life Technologies, Carlsbad, CA, USA) supplemented with 5\% newborn calf serum (NCS, S0750-500; Biowest SAS, Nuaillé, France) and 0.02 Amour units $/ \mathrm{ml}$ follicle stimulating hormone (FSH; Antrin 
R10; Kyoritsu Seiyaku Co., Tokyo, Japan) and then cultured in IVM medium under paraffin oil (Paraffin liquid, Nacalai Tesque Inc., Kyoto, Japan) (20 COCs per $100 \mu \mathrm{l}$ droplet) in 35-mm Petri dishes (351008, BD Falcon, Tokyo, Japan) under a humidified atmosphere of $5 \% \mathrm{CO}_{2}$ in air at $38.5{ }^{\circ} \mathrm{C}$ for $20-21 \mathrm{~h}$.

\section{Liquid preservation of oocytes}

At 20-21 h after the start of IVM, COCs were washed twice in the base medium which consisted of TCM-199 medium (Medium 199 powder, 31100-035, Gibco, Thermo Fisher Scientific) buffered with $11 \mathrm{mM}$ of HEPES (Sigma-Aldrich Co., St. Louis, MO, USA), 9 mM of Na-HEPES (Sigma-Aldrich Co.), and supplemented with $5 \mathrm{mM}$ of sodium bicarbonate with a $\mathrm{pH}$ adjusted to 7.3 and the osmolality to $0.290 \mathrm{osm}$. Then they were transferred into different containers in $1.3 \mathrm{ml}$ of storage medium according to the experimental design (described below). The storage medium was base medium supplemented with $10 \%(\mathrm{v} / \mathrm{v}) \mathrm{NCS}$ and $10.27 \mathrm{mM}$ sodium pyruvate (Suttirojpattana et al., 2017). The containers were then closed, sealed air tight with parafilm and kept at $25^{\circ} \mathrm{C}$ for $20 \mathrm{~h}$.

IVF

A straw of frozen Japanese Black bull semen was thawed by immersion in water at $37{ }^{\circ} \mathrm{C}$ for $40 \mathrm{sec}$. Then the sperm was loaded on the top of $3 \mathrm{ml}$ of $90 \%$ Percoll (Sigma-Aldrich Co., St. Louis, MO, USA) solution in a centrifuge tube and then centrifuged at $740 \times \mathrm{g}$ for $10 \mathrm{~min}$. The pellet was resuspended in $5.5 \mathrm{ml}$ of IVF 100 medium (IFP9630, Research Institute for Functional Peptides Co., Ltd., Yamagata, Japan) and centrifuged again at $540 \times g$ for $5 \mathrm{~min}$. The pellet was resuspended in IVF 100 medium to adjust the final sperm concentration to $3 \times 10^{6} \mathrm{sperm} / \mathrm{ml}$. Groups of 20 COCs were washed twice in IVF 100 medium and co-cultured with sperm under humidified atmosphere of $5 \% \mathrm{CO}_{2}$ in air for $4 \mathrm{~h}$ at $38.5^{\circ} \mathrm{C}$.

In vitro embryo culture (IVC)

After IVF, the presumptive zygotes were completely denuded from cumulus cells by gentle pipetting with a fine glass pipette in a culture medium which was Charles Rosenkrans 1 (CR1) medium (Rosenkrans et al., 1993) supplemented with amino acids (CR1aa) (Imai et al., 2002) and 5\% NCS. Then, 15-20 zygotes were washed and cultured in each $100 \mu 1$ droplet of culture medium under a humidified atmosphere of $5 \% \mathrm{CO}_{2}, 5 \% \mathrm{O}_{2}$, and $90 \% \mathrm{~N}_{2}$ at $38.5^{\circ} \mathrm{C}$ for up to 9 days. The day of IVF was considered as day 0 .

\section{Evaluation of blastocyst cell number}

On day 9 , embryos at or beyond the blastocyst stage were fixed in $99.5 \%$ ethanol supplemented with $10 \mu \mathrm{g} / \mathrm{ml}$ Hoechst 33342 (Calbiochem) overnight at 
$4{ }^{\circ} \mathrm{C}$. Then the embryos were mounted on glass slides in glycerol droplets. The total nuclei were counted under UV light with excitation at $330-385 \mathrm{~nm}$ and emission at $420 \mathrm{~nm}$ under an epifluorescence microscope (Nikon Eclipse E600, Tokyo, Japan).

\section{Experimental design}

Experiment 1 investigated the effect of different containers for the storage of matured oocytes on their subsequent developmental competence after IVF. Five different containers were compared, which were 1.5-ml closed (Eppendorf) tubes (ET) made of either polypropylene (PP, 0030 120.086, Eppendorf, Tokyo, Japan) (ET-PP group) or polystyrene (PS, 53071, Active Motif, Tokyo, Japan) (ET-PS group), 5-ml disposable TCT made of either PP (352063, BD Falcon, Tokyo, Japan) (TCT-PP group), or PS (352058, BD Falcon, Tokyo, Japan) (TCT-PS, group) or glass (9831-1207, Iwaki Glass Co., Tokyo, Japan) (TCT-G group). After IVM, groups of COCs were placed in storage medium in each type of container and stored for $20 \mathrm{~h}$ at $25^{\circ} \mathrm{C}$ as described above. After storage, the COCs were subjected to IVF and IVC as described above. Cleavage rates, blastocyst formation rates, rates of hatching and hatched blastocysts and blastocyst cell numbers were recorded on day 2, days 7-9, days 8-9 and day 9, respectively. For each oocyte lot, a group of matured COCs without storage was used as a control group. Five biological replicates were performed.

Experiment 2 investigated the effects of resveratrol during storage on developmental competence of matured oocytes. After IVM, COCs were stored for $20 \mathrm{~h}$ at $25^{\circ} \mathrm{C}$ in a glass TCT as described above in a serum-free storage medium supplemented with or without resveratrol at different concentrations $(0,0.1,1$ and $10 \mu \mathrm{M})$. In this experiment, the glass TCT was used according to the results of Experiment 1, and serum was omitted from the storage medium in order to avoid any interactions of non-defined factors with resveratrol. After storage, the oocytes were subjected to IVF and IVC as described above. Cleavage rates, blastocyst formation rates, rates of hatching and hatched blastocysts and blastocyst cell numbers were recorded on day 2, days 7-9, days 8-9 and day 9, respectively. A group of matured oocytes without storage was used as a control group. Five biological replicates were performed.

\section{Statistical analysis}

In all experiments, data were expressed as mean \pm SEM. The percentage data were arcsine transformed and analysed with one-way ANOVA; post-hoc Fisher's protected least significant difference test was used to locate differences. Significance was defined as $\mathrm{P}<0.05$. The entire statistical analysis was carried out using the software SPSS 20.0 for Windows (IBM Corp., Armonk, NY, USA). 


\section{Results}

Experiment 1: Effect of different containers during oocyte storage on subsequent embryo development. The mean percentage of cleaved oocytes was similar among all groups, ranging between $68.6-79.3 \%$. The oocytes without storage showed a significantly higher percentage of blastocyst formation compared with the storage groups (Table 1). Among the TCT groups the highest blastocyst rate was observed in the TCT-G group which was significantly higher than the rates in both the TCT-PP and TCT-PS groups but did not differ significantly from those of the ET-PP and ET-PS groups. There was no significant difference in hatching ability or total cell numbers of blastocysts among any of the groups.

Experiment 2: The effects of resveratrol during oocyte storage on subsequent embryo development. As shown in Table 2, the percentages of cleavage were similar among the control and stored groups; however, the blastocyst formation rate on day 9 in the control group was significantly higher $(\mathrm{P}<0.05)$ than in the stored groups, irrespective of resveratrol addition treatments during oocyte storage. The hatching ability and total cell numbers of blastocysts were not significantly different between the resveratrol-treated groups and the control group.

\section{Discussion}

In previous studies, liquid storage of oocytes was performed in Petri dishes or 4-well culture plates ( $\mathrm{Li}$ et al., 2012; Wang et al., 2014b; Zhao et al., 2015). Nevertheless, the surface coating of culture dishes may interact with the biological activities of animal cells such as those of embryos (Kolahi et al., 2012; Rivera and Rinaudo, 2012). Closed tubes seem to be more practical than Petri and culture dishes for oocyte storage under field or farm conditions. We tested five different tubes which differed in terms of type/size and material. Among the oocytes stored in TCT tubes, those stored in glass tubes showed significantly higher blastocyst development than those which were stored in plastic TCT tubes. This indicates that among the investigated materials, glass was superior for this purpose. This result is in accordance with previous observations on porcine embryos; when cultured in $5 \mathrm{ml}$ tubes, in vitro produced embryos showed the highest development rate to the blastocyst stage in glass tubes, whereas polypropylene caused retarded development (M. Ozawa, personal communication). Hence, disposable glass tubes could be successfully used for the culture of porcine embryos (Ozawa et al., 2006).

PP has been used for decades as a material for vessels to store different types of animal cells without obvious negative effects on their viability (Conning and Firth, 1969). However, in recent decades, the most conventional material for embryo culture is considered to be PS which withstands the temperature and humidity of the incubator without changing media characteristics (Smith et al., 2012; 
Blomfield et al., 2016). Our results indicated that compared with glass, both PP and PS exerted negative effects on the developmental competence of oocytes during storage. The toxicity of plastic materials and surface coatings is an issue that should be taken into account when oocytes are stored in media. Several synthetic polymers have been evaluated for compatibility with the development of mammalian embryos and not all types of polymers were found to be appropriate (Hunter et al., 1988). Certain products may carry water-soluble contaminants which alter the functions of animal cells and this effect can vary among production lots (Reuhl et al., 1990). Other studies indicated differences in material surface properties which affect cell behaviour, influencing experimental results during in vitro culture of somatic cells (Lau and Tchao, 2007; König et al., 2013; Zeiger et al., 2013). Nevertheless, the exact factor causing the difference in embryo developmental competence of oocytes stored in glass and PP tubes remains unclear. In the present study, development of oocytes stored in either 1.5-ml Eppendorf tubes or 5-ml culture test tubes did not differ significantly. Nevertheless, Eppendorf tubes are smaller and their fixed lids allow easier and safer logistics of stored oocytes compared to $5-\mathrm{ml}$ tubes. Based on the results presented above, a 1.5- or 2.0-ml glass tube with a safety lid may be the ideal vessel for this purpose. In the second experiment, we stored oocytes in the presence or absence of resveratrol using glass containers, based on the results of the first experiment. The aging of oocytes affects their quality through the increase of intracellular ROS to excessively high levels (Goud et al., 2008). Accordingly, storage of matured bovine oocytes for $20 \mathrm{~h}$ significantly reduces intracellular concentrations of GSH which controls the redox status in oocytes (Suttirojpattana et al., 2016). Acting as an antioxidant, resveratrol improves the development of cultured oocytes and embryos (Kwak and Hyun, 2012; Kwak et al., 2012; Liu et al., 2013; Wang et al., 2014a). In previous studies, resveratrol was reported to increase GSH concentrations in oocytes (Kwak et al., 2012; Wang et al., 2014a). Therefore, we hypothesised that resveratrol supplementation during oocyte storage may also be beneficial for embryo development after IVF. However, treatment of oocytes with resveratrol at different concentrations $(0.1,1$ and $10 \mu \mathrm{M})$ during oocyte storage affected neither the developmental rates to the blastocyst stage, nor the quality of blastocysts. The possible reasons may be that either (1) resveratrol was not potent enough to overcome the severe loss of GSH or (2) factors other than oxidative stress may contribute to the severe loss of developmental competence during oocyte storage. It is important to point out that the second experiment was conducted without the use of serum supplementation in the storage medium in order to avoid the possible interactions of serum-derived undefined factors with resveratrol. During storage, serum exerts positive effects on the developmental competence of matured oocytes (Suttirojpattana et al., 2017). Therefore, the lack of serum during oocyte preservation explains the lower blastocyst developmental rates and embryonic cell numbers in Experiment 2, compared with those of Experiment 1. 


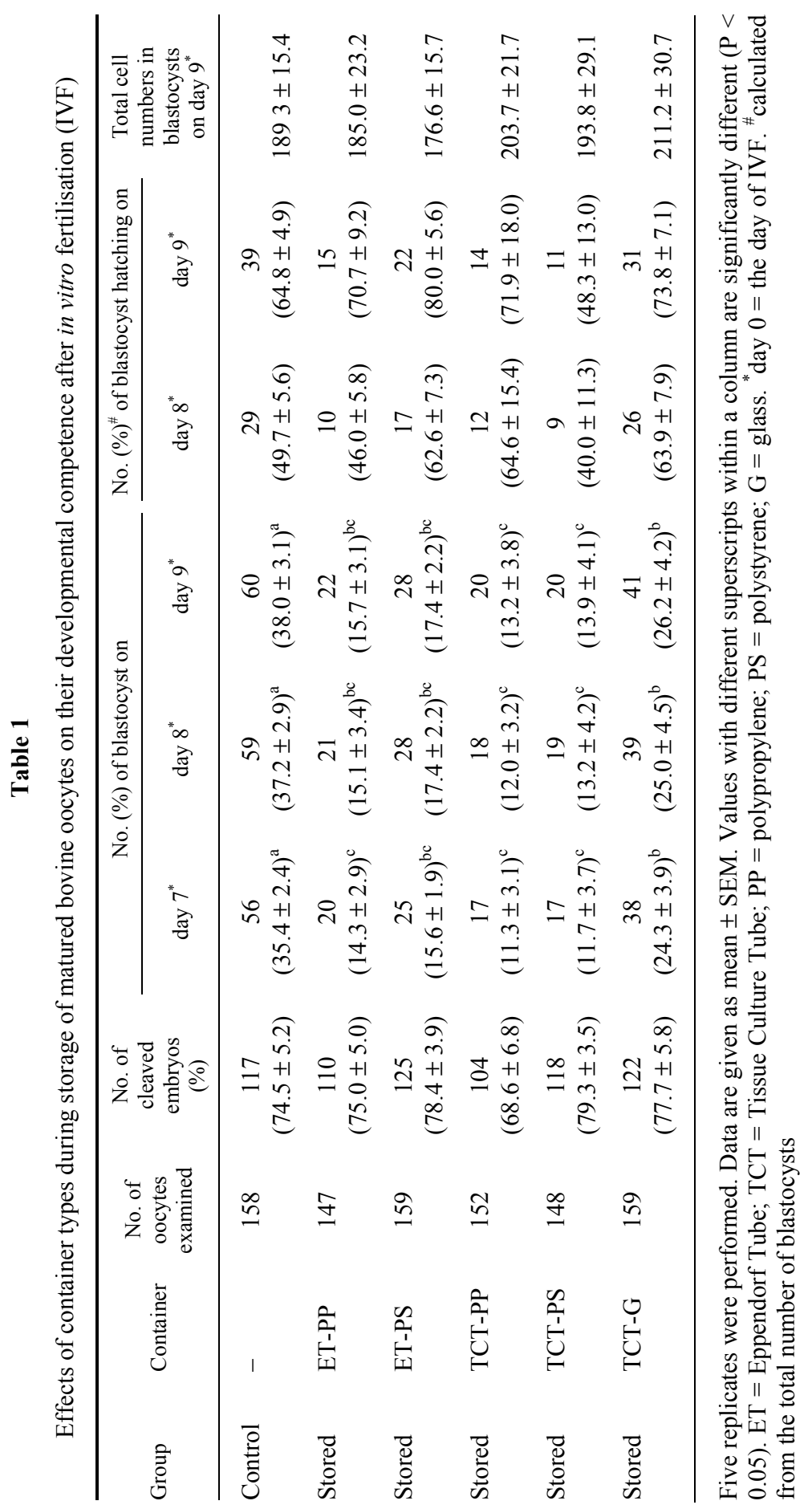




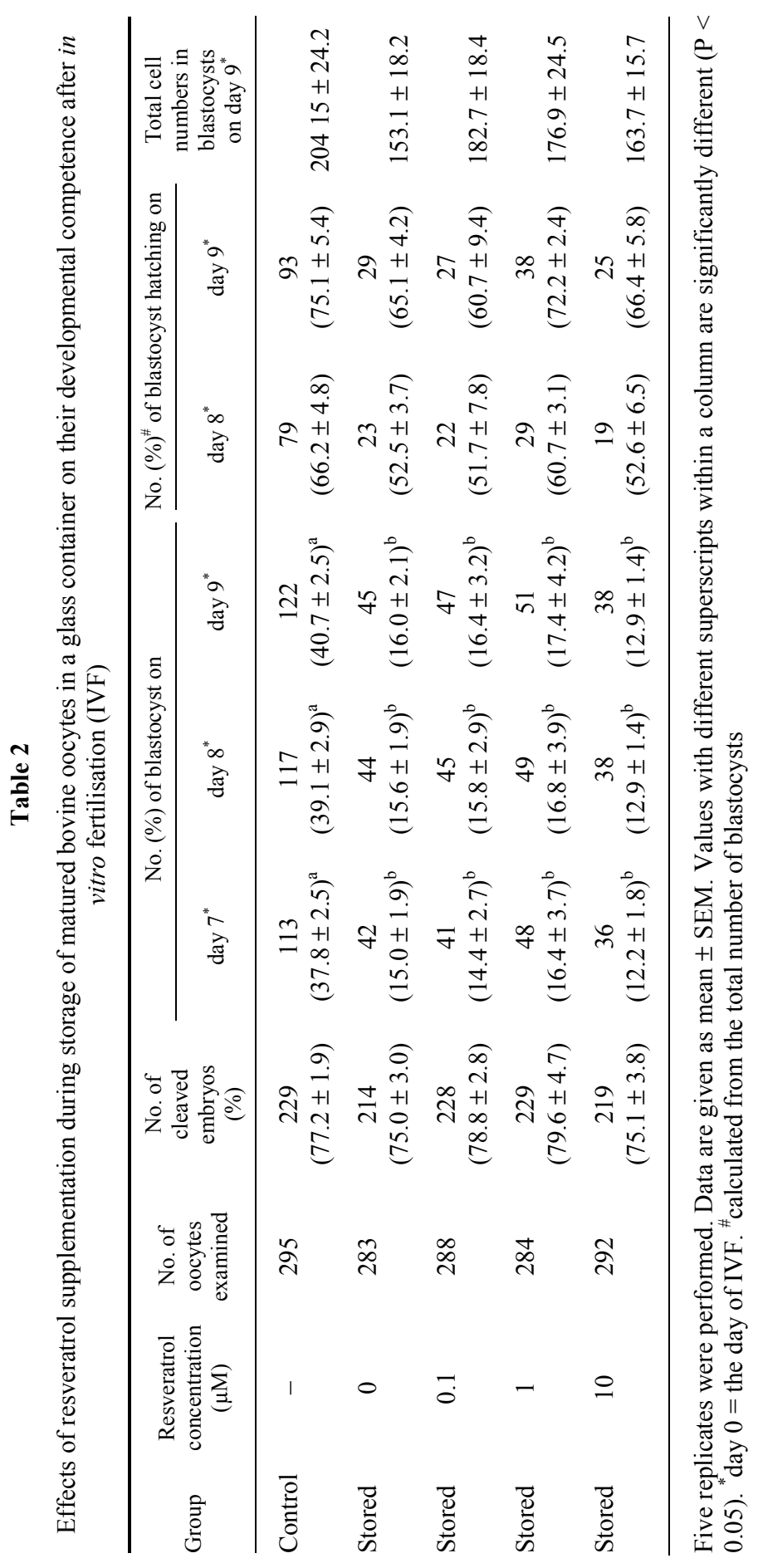


In conclusion, we found that glass is the optimum material for containers during oocyte storage and that medium supplementation with resveratrol did not affect the developmental competence of stored bovine oocytes. Further research will be required to improve the developmental competence of stored oocytes.

\section{Acknowledgements}

The study was financed by the NARO Institute of Livestock and Grassland Science, Japan (N32G4126). Tayita Suttirojpattana and Rangsun Parnpai were supported by the Royal Golden Jubilee PhD scholarship (2.B.TS/53/F.2).

\section{References}

Agung, B., Otoi, T., Wongsrikeao, P., Taniguchi, M., Shimizu, R., Watari, H. and Nagai, T. (2006): Effect of maturation culture period of oocytes on the sex ratio of in vitro fertilized bovine embryos. J. Reprod. Dev. 52, 123-127.

Blomfield, S., Redding, G. P., Bronlund, J. E., Steward, B. and Peek, J. (2016): A model for characterization of the thermal environment of embryos in petri dish during in vitro culture. Cell. Mol. Bioeng. 9, 546-555.

Conning, D. M. and Firth, J. (1969): Toxicity of polypropylene in tissue culture. Fd. Cosmet. Toxicol. 7, 461-472.

Fissore, R. A., Kurokawa, M., Knott, J., Zhang, M. and Smyth, J. (2002): Mechanisms underlying oocyte activation and postovulatory ageing. Reproduction 124, 745-754.

Goud, A. P., Goud, P. T., Diamond, M. P., Gonik, B. and Abu-Soud, H. M. (2008): Reactive oxygen species and oocyte aging: Role of superoxide, hydrogen peroxide and hypochlorous acid. Free Radic. Biol. Med. 44, 1295-1304.

Halldorsson, S., Lucumi, E., Gomez-Sjoberg, R. and Flemimg, R. M. T. (2015): Advantages and challenges of microfluidic cell culture in polydimethylsiloxane devices. Biosens. Bioelectron. 63, 218-231.

Hunter, S. K., Scott, J. R., Hull, D. and Urry, R. L. (1988): The gamete and embryo compatibility of various synthetic polymers. Fertil. Steril. 50, 110-116.

Imai, K., Matoba, S., Dochi, O. and Shimohira, I. (2002): Different factors affect developmental competence and cryotolerance in in vitro produced bovine embryo. J. Vet. Med. Sci. 64, 887-891.

Kolahi, K. S., Donjacour, A., Liu, X., Lin, W., Simbulan, R. K., Bloise, E., Maltepe, E. and Rinaudo, P. (2012): Effect of substrate stiffness on early mouse embryo development. Plos One 7, e41717.

Koyama, K., Sung-Sik, K., Huang, W., Yanagawa, Y., Takahashi, Y. and Nagano, M. (2014): Estimation of the optimal timing of fertilization for embryo development of in vitro-matured bovine oocytes based on the times of nuclear maturation and sperm penetration. J. Vet. Med. Sci. 76, 653-659.

König, J., Kohl, B., Kratz, K., Jung, F., Lendlein, A., Ertel, W. and Schulze-Tanzil, G. (2013): Effect of polystyrene and polyether imide cell culture inserts with different roughness on chondrocyte metabolic activity and gene expression profiles of aggrecan and collagen. Clin. Hemorheol. Microcirc. 55, 523-533.

Kwak, S. S. and Hyun, S. H. (2012): The effects of resveratrol on oocyte maturation and preimplantation embryo development. J. Embryo. Transf. 27, 71-80. 
Kwak, S. S., Cheong, S. A., Jeon, Y., Lee, E., Choi, K. C., Jeung, E. B. and Hyun, S. H. (2012): The effects of resveratrol on porcine oocyte in vitro maturation and subsequent embryonic development after parthenogenetic activation and in vitro fertilization. Theriogenology $\mathbf{7 8 ,}$ 86-101.

Lau, J. Y. and Tchao, R. (2007): Stressed polystyrene causes increased membrane sensitivity of adherent cells to fluid shear force: Technical note. Eur. Cell. Mater. 14, 40-44.

Li, Q., Wang, G., Zhang, J., Zhou, P., Wang, T. Y., Cui, W., Luo, M. J. and Tan, J. H. (2012): Combined inhibitory effects of pyruvate and low temperature on postovulatory aging of mouse oocytes. Biol. Reprod. 87, 1-11.

Liu, Y., He, X. Q., Huang, X., Ding, L., Xu, L., Shen, Y. T., Zhang, F., Zhu, M. B., Xu, B. H., Qi, Z. Q. and Wang, H. L. (2013): Resveratrol protects mouse oocytes from methylglyoxalinduced oxidative damage. Plos One 8, e77960.

Long, C., Damiani, P., Pinto-Correia, C., MacLean, R., Duby, R. and Robl, J. (1994): Morphology and subsequent development in culture of bovine oocytes matured in vitro under various conditions of fertilization. J. Reprod. Fertil. 102, 361-369.

Mullen, S. F. and Fahy, G. M. (2012): A chronologic review of mature oocyte vitrification research in cattle, pigs, and sheep. Theriogenology 78, 1709-1719.

Ozawa, M., Nagai, T., Kaneko, H., Noguchi, J., Ohnuma, K. and Kikuchi, K. (2006): Successful pig embryonic development in vitro outside a $\mathrm{CO}_{2}$ gas-regulated incubator: effects of $\mathrm{pH}$ and osmolality. Theriogenology 65, 860-869.

Reuhl, T. O. K., Amador, M. and Dani, J. A. (1990): Tissue culture tube contaminant inhibits excitatory synaptic channels. Brain Res. Bull. 25, 433-435.

Rivera, R. M. and Rinaudo, P. (2012): Bovine preimplantation embryo development is affected by the stiffness of the culture substrate. Mol. Reprod. Dev. 80, 184.

Rosenkrans, C., Zeng, G., McNamara, G., Schoff, P. and First, N. (1993): Development of bovine embryos in vitro as affected by energy substrates. Biol. Reprod. 49, 459-462.

Smith, G. D., Takayama, S. and Swain, J. E. (2012): Rethinking in vitro embryo culture: New developments in culture platforms and potential to improve assisted reproductive technologies. Biol. Reprod. 86, 1-10.

Suttirojpattana, T., Somfai, T., Matoba, S., Nagai, T., Parnpai, R. and Geshi, M. (2016): The effect of temperature during liquid storage of in vitro-matured bovine oocytes on subsequent embryo development. Theriogenology 85, 509-518.

Suttirojpattana, T., Somfai, T., Matoba, S., Nagai, T., Parnpai, R. and Geshi, M. (2017): Effect of medium additives during liquid storage on developmental competence of in vitro matured bovine oocytes. Anim. Sci. J. 88, 231-240.

Viana, J. H. M., Siqueira, L. G. B., Palhão, M. P. and Camargo, L. D. A. (2010): Use of in vitro fertilization technique in the last decade and its effect on Brazilian embryo industry and animal production. Acta Sci. Vet. 38, 661-674.

Wang, F., Tian, X. Z., Zhang, L., He, C. J., Ji, P. Y., Li, Y., Tan, D. X. and Liu, G. S. (2014a): Beneficial effect of resveratrol on bovine oocyte maturation and subsequent embryonic development after in vitro fertilization. Fertil. Steril. 101, 577-586.

Wang, T. Y., Li, Q., Li, Q., Li, H., Zhu, J., Cui, W., Jiao, G. Z. and Tan, J. H. (2014b): Non-frozen preservation protocols mature mouse oocytes dramatically extend their developmental competence by reducing oxidative stress. Mol. Hum. Reprod. 20, 318-329.

Zeiger, A. S., Hinton, B. and Van Vliet, K. J. (2013): Why the dish makes a difference: Quantitative comparison of polystyrene culture surfaces. Acta. Biomaterialia 9, 7354-7361.

Zhao, S., Liu, Z. X., Bao, Z. J., Wu, Y., Wang, K., Yu, G. M., Wang, C. M. and Zeng, S. M. (2015): Age-associated potency decline in bovine oocytes is delayed by blocking extracellular $\mathrm{Ca}^{2+}$ influx. Theriogenology 83, 1493-1501. 